\title{
LES EMPIDIDAE HEMERODROMIINAE D'AUTRICHE
}

\section{[Diptera]}

par F. VaILlant.

Trente-neuf espèces européennes d'Empididae Hemerodromiinae sont connues d'Autriche; ce sont :

Phyllodromia melanocephala (FABR.), Chelipoda albiseta (ZETT.), Ch. vocatoria (FALx.), Hemerodromia albicornis MeIgen, H. oratoriaoratoria (FALL.), H. unilineata ZETT., Chelifera flavella (ZETT.), Ch. precatoria (FALL.), Ch. trapezina (ZETT.), Metachela nigriventris (LoEw), Dolichocephala guttata (HAL.), D. irrorata (FALLÉN), Clinocera appendiculata (ZETT.), Cl. appendiculata-storchi Mıк, Cl. bipunctata (HAL.), Cl. fontinalis $\mathrm{HAL}_{\mathrm{AL}}, \mathrm{Cl}$. inermis (LoEw), Cl. multiseta Strorl, Cl. nigra (Loew), Cl. nudipes (LoEw), Cl. plectrum (MIK), Cl. stagnalis (HALIDAY), Cl. tibiella (MIK), Cl. varipennis (NowickI), Cl. wesmaeli (MAcQ.), Wiedemannia aquilex (LoEw), W. beckeri-beckeri Mrk, W. bistigma (Curtis), W. bohemani (Zetr.), W. erminea Mı, W. escheri (Zетт.), W. fallaciosafallaciosa (L.), W. fallaciosa-impudica (Mı), W. hygrobia (Loew), W. lamellata (LoEw), W. mikiana Bezzr, W. phantasma (MrK), W. pirata (MIK), W. rhynchops-austriaca VAILLANT, W. wachtli (MIK), W. zetterstedti (FALLÉN).

Malheureusement, G. STrobl et les autres entomologistes qui ont capturé ces insectes n'ont donné, le plus souvent, aucun renseignement sur leurs biotopes.

En 1959 et en 1960, j'ai effectué deux séjours en Autriche, et en ai profité pour capturer quelques Hemerodromiinae; il m'a paru utile de donner une liste des exemplaires recueillis et de fournir des précisions concernant les caractères de leurs habitats :

Dolichocephala austriaca VaIllant : La Schwarza, près d'Hirschwang (Raxalpe, Basse Autriche), 25-VIII-1960, $500 \mathrm{~m}$ d'altitude, $1 \hat{o}$ sur une paroi moussue suintante. Cette espèce est également connue des Pyrénées.

Clinocera (Clinocera) appendiculata ZETTERSTEDT : Hallstatt (Haute Autriche), $510 \mathrm{~m}, 3-\mathrm{IX}-1960$, de nombreux exemplaires sur des roches dolomitiques suintantes. La Kapuzinerberg à Salzburg, $230 \mathrm{~m}$, le 14-VIII-1960, sur un mur suintant, nombreux spécimens. 
Clinocera (Kowarzia) plectrum (Мıк) : Kanzelhöhe (Carinthie), $1400 \mathrm{~m}$, le 29-VIII-1960, 1 ô sur une pierre en bordure d'une source.

Clinocera (Clinocera) schremmeri (VallLaNT) : Col du Hochtor (Hohe Tauern, vers $2400 \mathrm{~m}$ ); une larve recueillie, le 2-IX-1960, dans la mousse d'un ruisseau à pente rapide, donna une imago $\hat{o}$ le 20-IX-1960. Gerlitzen (Carinthie, $1900 \mathrm{~m}$ ), 2 o sur des rochers suintants. Près du sommet de la Wollanernock (Carinthie, $2090 \mathrm{~m}$ ), 1 ô dans un habitat du même type.

Clinocera (Kowarzia) tibiella (Мик) : Tous les exemplaires ont été recueillis sur des parois rocheuses suintantes. Ruisseau se déversant dans l'Attersee (Haute Autriche, $470 \mathrm{~m}$ ), le 31-VIII-1959, 1 s. Ruisseau affluent du lac de Millstatt (Carinthie, $580 \mathrm{~m}$ ), 5-IX-1960, nombreux exemplaires. Au-dessus de Feld-am-Brennsee (Carinthie, $900 \mathrm{~m}$ ), le 1-IX-1960, nombreux exemplaires. Kanzelhöhe (Carinthie, $1400 \mathrm{~m}$ ), le 29-VIII-1960, 3 o.

Wiedemannia (Wiedemannia) bistigma (CurTIs) : Sur des blocs granitiques dans un ruisseau près de Mauthausen (Haute Autriche, $230 \mathrm{~m}$ ), le 16-VIII-1960, 2 t et 1 i.

Wiedemannia (Philolutra) bohemani (ZETT.) : Partie torrentueuse de la Schwarza, près d'Hirschwang, $550 \mathrm{~m}$, le 26-VIII-1960, de nombreux exemplaires sur les blocs battus par l'eau.

Wiedemannia (Eucelidia) escheri (ZETr.) : Sur les pierres d'un ruisseau dans la Wienerwald, vers $250 \mathrm{~m}$, le 24-VIII-1960, 2 o. Sur des blocs granitiques dans un ruisseau près de Mauthausen (Haute Autriche, $230 \mathrm{~m}$ ), le 16-VIII-1960, 2 ㅇ.

Wiedemannia (Philolutra) fallaciosa (LoEw) : Ruisseau affluent du lac de Millstatt (Carinthie, $580 \mathrm{~m}$ ), le 5-IX-1960, 3 o et 5 우 sur les plus grosses pierres du ruisseau.

Wiedemannia (Pseudowiedemannia) lamellata (LoEw) : Le Danube à Linz, en Haute Autriche, $265 \mathrm{~m}$, le 16-VIII-1960, 2 \& et 1 o sur les pierres du quai dans la partie mouillée par capillarité et battue par le flot.

Wiedemannia (Camelopis) phantasma (MIK) : La Drau à Villach (Carinthie, $501 \mathrm{~m}$ ), le 30-VIII-1960, 2 o et 1 \%.

Wiedemannia (Wiedemannia) rhynchops austriaca VAILLANT : Important ruisseau entre Neumarkt in Steiermark et Wildbad Einöd, $830 \mathrm{~m}$, le 27-VIII-1960. 


\section{DISCUSSION}

Quarante et une espèces d'Hemerodromiinae sont représentéee en Autriche; la plupart ont une vaste répartition en Europe et leur aire géographique comprend les Alpes Occidentales. Toutefois Clinocera varipennis n'est connue que des Alpes Orientales et des Carpathes; Wiedemannia escheri et $W$. pirata, qui sont probablement l'une et l'autre des espèces de régions peu élevées, auraient leur aire limitée aux montagnes de très faible altitude bordant la vallée du Danube, entre l'Autriche et la Mer Noire. Dolichocephala austriaca est représentée en Autriche, ainsi que dans les Pyrénées, et elle l'est probablement aussi dans les Alpes Occidentales. Seules Clinocera multiseta et Wiedemannia erminea seraient endémiques des Alpes Orientales.

Wiedemannia bistigma accompagne, en France, certains cours d'eau lents de plaine, mais des exemplaires n'avaient été trouvés, jusqu'ici, que dans des rivières importantes. Or cette espèce semble s'accommoder, près de Mauthausen, de minuscules ruisseaux.

Clinocera schremmeri semble, dans les Alpes Orientales, localisée dans les parties hautes, au-dessus de $1900 \mathrm{~m}$, alors que l'espèce voisine $\mathrm{Cl}$. appendiculata est commune à Salzbourg et n'a jamais été trouvée en altitude. Or, dans les Alpes du Dauphiné, cette dernière espèce ne semble pas représentée en dessous de $1200 \mathrm{~m}$ et son aire s'étend jusqu'à $2200 \mathrm{~m}$; inversement, $C l$. schremmeri est encore commune vers $800 \mathrm{~m}$.

\section{RÉSUMÉ}

L'auteur fait une révision des Diptères de la sous-famille des Empididae Hemerodromiinae pour l'Autriche et montre que la plupart des espèces figurent dans l'ensemble des Alpes.

\section{THE EMPIDIDAE HEMERODROMINAE FROM AUSTRIA}

[Diptera]

The author revises the Diptera of the subfamily Empididae Hemerodromiinae from Austria and points out that the range of almost all the species extends both to the eastern and to the western Alps.

\section{DIE EMPIDIDAF HEMERODROMINAE IN ÖSTERREICH [Diptera]}

Der Verfasser unterzicht die in österreich vorkommenden Dipteren der Unterfamilie der Empididae Hemerodromiinae einer neuen Betrachtung und zeigt, dass die meisten Arten in den ganzen Alpen vorhanden sind. 


\section{TRAVAUX CITÉS}

Engel (0.). 1938-1940. - in E. Lindner, Die Fliegen der Palaearktischen Region, II, 4, Stuttgart.

Kertesz (C.). 1908. - Catalogus Dipterorum, III.

Pomeisl (E.). 1953. - Studien an Dipterenlarven des Mauerbaches; Beiträge zur Limnologie der Wienerwaldbäche. Wetter und Leben. $165-176$.

Schiner (R.). 1860. - Fauna Austriaca. Die Fliegen (Diptera), Wien. Strobl (G.). 1910. - Dipteren von Steiermark, II. Nachtrag. Mitteil. Naturwiss. ver. f. Steiermark, 46 : 57-86.

Vaillant (F.). 1964. - Révision des Empididae Hemerodromiinae de France, d'Espagne et d'Afrique du Nord (Dipt.). Ann. Soc. Ent. Fr., 133 : 143-171.

Vaillant (F.). 1967. - in J. Illies : Limnofauna Europaea, Stuttgart.

VAILLANT (F.). 1967. - La répartition des Wiedemannia dans les cours d'eau et leur utilisation comme indicateurs de zones écologiques (Diptera, Empididae). Annls Limnol., 3 : 267-293.

Vaillant (F.). 1968. - Quelques Empididae Hemerodromiinae de Pologne (Diptera). Fragm. Faunystica, Warszawa.

(Laboratoire de Zoologie, Faculté des Sciences, 38 - Saint-Martin-d'Hères.) 